\title{
Entertainment Modeling in Physical Play Through Physiology Beyond Heart-Rate
}

\author{
Georgios N. Yannakakis and John Hallam \\ Maersk Mc-Kinney Moller Institute \\ University of Southern Denmark \\ Campusvej 55, Odense M, DK-5230 \\ \{georgios, john\}@mmmi.sdu.dk
}

\begin{abstract}
An investigation into capturing the relation of physiology, beyond heart rate recording, to expressed preferences of entertainment in children's physical gameplay is presented in this paper. An exploratory survey experiment raises the difficulties of isolating elements derived (solely) from heart rate recordings attributed to reported entertainment and a control experiment for surmounting those difficulties is proposed. Then a survey experiment on a larger scale is devised where more physiological signals (Blood Volume Pulse and Skin Conductance) are collected and analyzed. Given effective data collection a set of numerical features is extracted from the child's physiological state. A preference learning mechanism based on neuro-evolution is used to construct a function of single physiological features that models the players' notion of 'fun' for the games under investigation. Performance of the model is evaluated by the degree to which the preferences predicted by the model match those expressed by the children. Results indicate that there appears to be increased mental/emotional effort in preferred games of children.
\end{abstract}

\section{Introduction}

The principal goal in the reported work is to construct a user model of a class of game-playing experience. Specifically, the aim is that the model can predict the answers to which variants of a given game are more or less "fun." This approach is referred to as Entertainment Modeling. Entertainment generated by a physical game experience is captured through features extracted from the player's physiological state and feature selection is used for choosing the appropriate set of features that successfully predict expressed entertainment preferences. Game play experiences might very well be identified through features extracted from user-game interaction. Furthermore, game play behavior could be video recorded and emotions could be recognized by experts or automatically through face gesture detection; however, these approaches are not the focus of this work.

In this work the entertainment model is constructed using preference learning techniques applied to statistical features derived from physiological signals measured during play. The output of the constructed model is a real number in the range $[0,1]$ such that more enjoyable games receive higher numerical output. This basic approach of entertainment modeling is applicable to a variety

A. Paiva, R. Prada, and R.W. Picard (Eds.): ACII 2007, LNCS 4738, pp. 256-267 2007.

(C) Springer-Verlag Berlin Heidelberg 2007 
of games, both computer [1] and physical [23/4] using features derived from physiological data and/or from the interaction of player and opponent measured through game parameters.

Even though entertainment is a highly complicated mental state it is correlated with sympathetic arousal [5] which can be captured through specific physiological signals such as heart rate variability (HRV) and skin conductivity [6]. While the emotional impact on a subject's physiological state during computer game playing is well reported in the literature (see [5 among others), there are no corresponding studies in the physical play domain. Motivated by the lack of entertainment modeling approaches grounded on a player's physiological state in physical interactive games, the Playware [7] physical interactive game platform has been used for recoding Heart Rate (HR) signals of children during play [8]3. Herein, results and conclusions derived from this exploratory experiment (56 children participants) are presented. The complexity of isolating the elements of physical activity from expressed entertainment in physical games is outlined through this experiment. This problem is handled, in part, through a carefully designed control experiment of physical activity reported in [4.

As a sequel to the exploratory experiment, a new set of experiments for capturing entertainment preferences through physiology in physical play is presented here. This experiment expands the investigation of the physiological state's relation to entertainment preferences from HR to Blood Volume Pulse (BVP) and Skin Conductance (SC) signals. Moreover, the number of child participants is increased to 72 allowing for safer conclusions given our aims. A statistical analysis reveals that features extracted from HR and BVP that correspond to both physical and mental/emotional effort correlate significantly with expressed preferences. Moreover, preference learning (neuro-evolution) attempts on single features indicate that the energy of the high frequency band (derived through power spectral analysis) of HRV constitutes the feature that performs best in predicting expressed preferences on unknown data. This feature, which is suppressed during mental or emotional stress [9]10, is highly anti-correlated to reported entertainment indicating high parasympathetic heart activity on preferred games. Results also suggest that collecting physiological signals beyond $\mathrm{HR}$, such as BVP, may provide more meaningful features for capturing entertainment preferences of children in physical play.

\section{Related Work}

Physiological condition measures have been used extensively for emotion recognition in children and adults within the affective computing research area. Mandryk et al. [5] examine the correlations between physiological signals (galvanic skin response, electromyography in jaw, respiration and cardiovascular measures) in reported adult user experiences in computer games. The preliminary experiments of Rani et al. 11] for appropriately adjusting the level of challenge in the game of 'Pong' based on recorded physiological signals in realtime and subject's self-reports of their emotional experiences during gameplay 
is closely related to our work. That study, however, is primarily focused on the anxiety level detection in real-time and is limited by the number of human participants. Physiological state (HR, SC) prediction models have also been proposed for potential entertainment augmentation in computer games [12.

Working on the same basis as Mandryk et al. [5], Ravaja et al. [13] examined whether the nature of the game opponent influences the physiological state of players. In addition, Hazlett's [14] work is focused on the use of facial electromyography to distinguish positive and negative emotional valence during interaction with a racing video game.

All aforementioned studies are focused on the use of physiology for capturing user experiences (e.g. "fun", engagement or excitement) applied within the computer and edutainment games framework. The work reported here is novel in that it examines the physiological state (HR, BVP, SC) correlates of reported "fun" in physical activity games, attempts to isolate physiological signal features attributed to reported entertainment in such physically demanding games and proposes a way of constructing a subjective model (a predictor of user preferences) of reported "fun" grounded in statistical features of physiological signal dynamics.

\section{Test-Bed Physical Games}

The Playware [7] prototype playground consists of several building blocks (i.e. tangible tiles) that allow for the game designer (e.g. the child) to develop a significant number of different games within the same platform. The overall technological concept of Playware is based on intelligent physical identities (tiles) that incorporate processing power, communication, input and output, focusing on the role of the morphology-intelligence interplay in developing game platforms [7/244.

Two games were designed on Playware and used for the experiments presented here: 'Bug-Smasher' and 'Space-Invaders'. Bug-Smasher is developed on a $6 \times 6$ square tile topology. During the game, different 'bugs' (colored lights) appear randomly on the game surface and disappear sequentially after a short period of time by turning a tile's light on and off respectively. The child's goal is to smash as many bugs as possible by stepping on the lighted tiles. Bug-smasher has been used as a test-bed in previous work; further details can been found in [2]8/4].

On the other hand, Space-Invaders' design is based on the classical arcade game released by Taito in 1978 . The game is implemented on a $5 \times 10$ tile topology. During the game, different alien spaceships (colored lights) appear on the top side of the game topology and move towards the bottom row of the game where the player's loaded 'guns' are placed. The child's goal is to shoot the alien spaceships down by firing at them. A shot is fired by pressing the lighted tiles indicating the guns. Further details on the Space-Invaders game can be found in 3.4.

\section{Experiment Setup}

According to the experimental design proposed in [4], the test-bed game under investigation is played in variants. For this purpose, different states (e.g. 'Low', 
'High') of quantitative estimators of qualitative entertainment factors (e.g. challenge) are used. The combination of states/number of entertainment factors generates a pool of dissimilar games for the designer to investigate.

By experimental design (see [12]), each subject plays against $k$ of the selected $n$ variants of the selected game in all permutations of pairs. ( $k$ equal 2 and $n$ equals 8 and 9 in the preliminary/exploratory and the main experiment respectively in this paper.) Thus, $C_{k}^{n}$ is the required number of subjects to cover all combinations of $k$ out of $n$ game variants. More specifically, each child plays games in pairs (game $A$ and game $B$ ) - differing in the levels/states of one or more of the selected entertainment factors - for a selected time window. Each time a pair of games ('game pair') is finished, the child is asked whether the first game was more "fun" than the second game (pairwise preference). Children are not interviewed but are asked to fill in a questionnaire, minimizing the interviewing effects reported in 5. To minimize any potential order effects we let each child play the aforementioned games in the inverse order too. Statistical analysis of the effect of order of game playing on children's judgement of entertainment indicates the level of randomness in children's preferences (see [4]). Randomness is apparent when there is a different preference in the pair $(A, B)$; i.e. $A \succ B$ and $B \succ A$.

All subjects are given the same instructions by an experimenter who is unaware of the purpose of the experiment, minimizing experimenter expectancy effects 15. The playing time window chosen (90 seconds in this paper) is a compromise between effective data collection (long enough subject-game interaction to support a relative judgement) and not overstretching children on excessive periods of energetic physical play.

Emotion, such as entertainment, capture is considered, in general, a hard problem mainly because understanding emotion is hard 16. Capturing reports of playing experiences or emotions is still tough since data obtained embed experimental noise and subjectivity. As previously mentioned, a pairwise preference scheme (2-alternative forced choice: 2 -AFC) is used in self-reports of children. 2-AFC offers several advantages for a subjective entertainment capture: it minimizes the assumptions made about subjects' notions of "fun" and allows a fair comparison between the answers of different humans. Since the focus is to construct a model relating reported entertainment preferences to individual playing features that generalizes over the reports of different players 2-AFC is preferred to a ranking approach [5. Forcing the choice of subjects generates experimental noise, in that the subject may have no significant preference for one or other of the game variants played yet must nevertheless express a preference; however, insignificant order effects provide evidence that the experimental noise generated in this way is random. Previous studies 2 have shown that reported fun cannot be captured solely on the basis of game variants since individuality (e.g. through physiological measures) is required for effectively modeling expressed preferences of entertainment.

Note that in the presented studies subjects played all their assigned games on the same day, mitigating day-dependence effects on their physiology [16]. 
Moreover, experiments held meet three of the five factors for eliciting genuine emotion in the most natural setup (ibid.): the experiments took place in a setup close to the real-world since children played in their school classroom, our emphasis was on internal feelings and subjects were not aware of the purpose of the experiment (other-purpose).

\section{Machine Learning}

The proposed approach to entertainment modeling is based on selecting a minimal subset of individual features and constructing a quantitative user model that predicts the subject's reported entertainment preferences. The assumption is that the entertainment value $y$ of a given game, which models the subject's internal response to playing the game, that is, how much "fun" it is, is an unknown function of individual features which a machine learning mechanism can learn. The subject's expressed preferences constrain but do not specify the values of $y$ for individual games but we assume that the subject's expressed preferences are consistent.

Any machine learning which is based on learning a target output is inapplicable since target outputs are unknown. By the use of a ranking approach numerical values for the $y$ variable could be made available; however, ranking is an undesired method for the self-report design of comparative "fun" analysis for the disadvantages mentioned earlier. Preference learning [17] is the only applicable type of machine learning for this constrained classification problem. There are several techniques that learn from a set of pairwise preferences such as algorithms based on support vector machines and perceptron modeling [18. However, given the high level of subjectivity of human preferences and the highly-noisy nature of input data, we believe that more complex non-linear functions such as Artificial Neural Networks (ANN) serve our purposes better. Thus, feedforward multilayered Neural Networks for learning the relation between the selected player features (ANN inputs) and the "entertainment value" (ANN output) of a game are used in the experiments presented here. Even though ANNs may achieve high training performance are not, in general, easily interpreted; however, a small (input) feature set and/or the inclusion of rules in the ANN structure (e.g. fuzzy-ANN [1) could make interpretation a much easier task [4. Since there are no prescribed target outputs for the learning problem (i.e. no differentiable output error function), ANN training algorithms such as back-propagation are inapplicable. Learning is achieved through artificial evolution. Details on the neuro-evolution mechanism used can be found in [3]. Other preference learning approaches are considered for comparison as a direction for future work.

\section{Exploratory Experiment}

Fifty six normal-weighted (based on their body mass index) children whose ages covered a range between 8 and 10 years participated in this exploratory experiment. The 56 children were split into two groups of 28 children and each group 
was assigned to play either Bug-Smasher or Space-Invaders according to the protocol presented in section 4 . To investigate the interplay between entertainment and physical activity we asked all 56 children to participate in an additional experiment: each child was asked to run around a $3 \mathrm{~m} \times 3 \mathrm{~m}$ space for 90 seconds. The assumption here is that this exercise (physical activity control) task is a non-entertaining activity for the child since children were not asked whether it was "fun" or not and it was not compared to any physical game task. Further details on the experimental protocol used can be found in [3].

In this experiment the following statistical parameters are extracted from HR signals recorded while children playing: the average HR $E\{h\}$, the standard deviation of $\mathrm{HR} \sigma\{h\}$, the maximum $\mathrm{HR} \max \{h\}$, the minimum $\mathrm{HR} \min \{h\}$, the correlation coefficient $R_{h}$ between HR recordings and the time $t$ in which data were recorded, the autocorrelation (lag equals 1 ) of the signal $\rho_{1}^{h}$ and the approximate entropy $\left(A p E n_{h}\right)[19$ of the signal which quantifies the unpredictability of fluctuations in the HR time series. In addition, three different regression models were used to fit (least square fitting) the HR signal: linear, quadratic and exponential. The additional features were the parameters of the three regression models mentioned above. The computation of HRV features was not possible given the data provided by the available recording apparatus since the detected RR intervals are opaquely converted into HR estimates by the wireless POLAR s610i ECG device used.

Statistical analysis showed that average HR appears to be the only feature examined that is significantly correlated to reported entertainment $(r=0.4146$, $\mathrm{p}=0.0057)$. This interplay between engagement, physical activity and entertainment demonstrated in 813 is consistent with the significant correlation between the average response time of children interacting with Playware games and reported entertainment 2]. (This is unsurprising, since one would expect a more enjoyable game to induce greater physical effort from the player.)

\subsection{Feature Selection and Preference Learning}

Given the selected features (ANN input) and the expressed preferences of entertainment ANNs are evolved by following the approach presented in [3] and evaluated through the leave-one-out cross-validation method. The two feature selection methods (n Best Feature Selection and Sequential Forward Selection) described in [3] are applied and compared. The initial subset (ANN input) for both methods includes the feature that performs best in the single feature experiment: ApEn $\underline{3}$.

The best cross-validation performance $(80.66 \%$; average of $88 \%, 78 \%$ and $76 \%)$ is achieved when the ANN input contains $A p E n$ and $E\{h\}$. More HR signal features added in the feature subset do not yield significantly higher classification accuracy [3. The relation between $A p E n, E\{h\}$ and the game's predicted entertainment value $(y)$ given by the highest performing ANN found is illustrated in Fig. 1. Note that the three fittest ANNs generated, each trained on different portions of $2 / 3$ of total data, exhibit the same qualitative features of the surface illustrated in Fig. 1. 


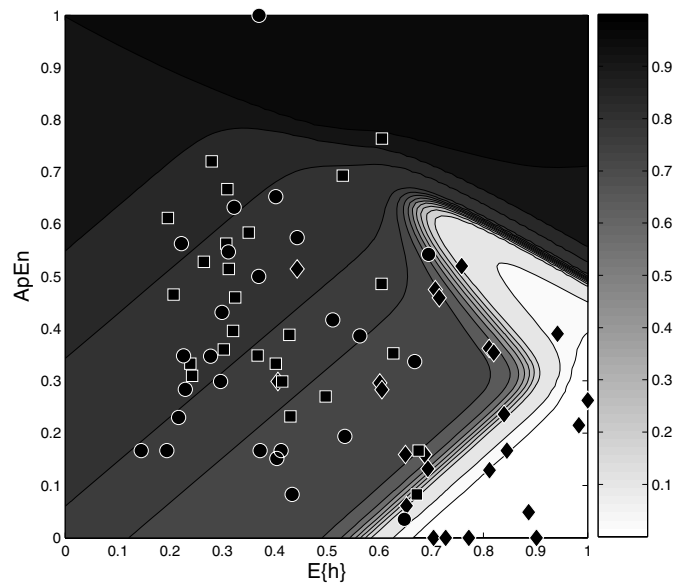

Fig. 1. Evolved ANN that yields the best classification accuracy on unknown data (88.00\%): ANN output $y$ (entertainment; the darker the higher) with regards to $E\{h\}$ and $A p E n$. Points plotted correspond to the 75 data of the validation set including 25 preferred (entertaining) games (squares), 25 non-preferred (non-entertaining) games (circles) and 25 exercise trials (diamonds).

According to Fig. 1, a physical activity is not entertaining when high $E\{h\}$ values $(E\{h\}>0.7)$ are combined with lower than average $A p E n$ values $(A p E n<$ $0.5)$. Given the experiments presented in this paper, this is a common situation in pure exercise physical activities which are not considered entertaining by children according to our assumption. Highly entertaining games are the ones that correspond to a combination of very high $E\{h\}$ and $A p E n$ values $(E\{h\}>0.6$, $A p E n>0.7)$.

HR signals obtained show that the running task appears to involve much more physical effort (high $E\{h\}$ values) than the physical effort required in a physical game, and further that the physical effort involved is different in kind (low $A p E n$ values; high regularity of the HR signal). It follows that the exercise/running control experiment may generate HR dynamics rather easy to separate from game-play HR dynamics, and allows one to distinguish entertaining game-play from exercise purely on the artificial basis of the kind of physical activity taking place. It is therefore, in retrospect, not a good control for physical activity effects in game play.

Thus, it is likely that constructed user models would be less effective for distinguishing more and less enjoyable games based on the degree rather than the kind of physical activity they engender. To deal with this and control for any elements of physical activity in the physiological measurements, an objectively (by human-verification) non-entertaining form of physical activity needs to be tested. Preliminary results have shown that user ANN models able to predict children's preferred game variants given suitable HR dynamics feature representations can indeed be constructed and that such models not only distinguish 
game-play from game-like non-entertaining physical activity but also generalize (to some extent) over children's individual preferences 4].

\section{Player's Physiology Beyond Heart-Rate}

This section presents an initial analysis of data collected through an additional larger survey experiment using the experimental protocol presented in section 4 . In this experiment physiology data collection expands to blood volume pulse and skin conductance signal recordings. The ProComp Infiniti biosensoring system by Thought Technology is used for this set of experiments. Seventy two children participated each playing a pair of variants of the Bug-Smasher game in both orders (see section 4).

The features extracted from the obtained signals include the fifteen statistical parameters of the HR signal presented in previous studies (see section 6 and [8]). The additional features for each signal type are as follows:

HR. The initial HR recording $h_{\text {in }}$, the last HR recording $h_{\text {last }}$, the time when maximum HR occurred $t_{\text {max }}^{h}$, the time when minimum HR occurred $t_{\text {min }}^{h}$, the difference $t_{\text {max }}^{h}-t_{\text {min }}^{h}$.

BVP. The average BVP $E\{b\}$, the standard deviation of BVP $\sigma\{b\}$, the maximum BVP $\max \{b\}$, the minimum BVP $\min \{b\}$, the average inter-beat amplitude $E\{I B A m p\}$, the mean of the absolute values of the first and second differences of the raw BVP [16] ( $\delta_{|1|}^{b}$ and $\delta_{|2|}^{b}$ respectively) and the following HRV parameters:

- HRV - time domain: the standard deviation of RR intervals $\sigma\{R R\}$, the fraction of RR intervals that differ by more than $50 \mathrm{msec}$ from the previous $R R$ interval $p R R 50$, the root-mean-square of successive differences of RR intervals $R M S_{R R}$ [10.

- HRV - frequency domain: the frequency band energy values derived from power spectra obtained using discrete Fourier transformation; energy values are computed as the integral of the power of each of the following four frequency bands (see [10]20] among others): Ultra Low Frequency (ULF) band: $[0.0,0.0033] \mathrm{Hz}$; Very Low Frequency (VLF) band: $(0.0033,0.04]$ Hz; Low Frequency (LF) band: $(0.04,0.15] \mathrm{Hz}$ and High Frequency (HF) band: $(0.15,0.4] \mathrm{Hz}$.

SC. All extracted features used for the HR signal. Additional features include the mean of the first and second differences of the raw SC $\left(\delta_{1}^{s}\right.$ and $\delta_{2}^{s}$ respectively) and the mean of the absolute values of the first and second differences of the raw $\mathrm{SC}\left(\delta_{|1|}^{s}\right.$ and $\delta_{|2|}^{s}$ respectively).

\subsection{Statistical Analysis}

The correlation coefficients between children's expressed preferences and recorded physiological signal features are obtained through $c(\vec{z})=\sum_{i=1}^{N_{s}}\left\{z_{i} / N_{s}\right\}$, where $N_{s}$ is the total number of game pairs where physiological signals were properly recorded $\left(N_{s}=115\right.$ for $\mathrm{HR}$ and BVP and $N_{s}=85$ for SC) and $z_{i}=1$, if 
the subject chooses as the more entertaining game the one with the larger value of the examined feature and $z_{i}=-1$, if the subject chooses the other game in the game pair $i$.

Table 1. Correlation coefficients between reported entertainment and individual physiological features. For reasons of space, the three highest absolute correlation coefficient values for each physiological signal type are ranked and presented here. $\gamma_{s}$ is the parameter of the quadratic regression $\left(s_{Q}(t)=\beta_{s} t^{2}+\gamma_{s} t+\epsilon\right)$ on the SC signal which quantifies the rotation angle with respect to the $\mathrm{x}$-axis of the quadratic curve. Statistically significant effects appear in bold.

\begin{tabular}{c|c||c|c||c|c}
\hline \hline HR & $c(\vec{z})$ & BVP & $c(\vec{z})$ & SC & $c(\vec{z})$ \\
\hline \hline$E\{h\}$ & $\mathbf{0 . 2 2 4}$ & $\delta_{|1|}^{b}$ & $\mathbf{0 . 2 1 6}$ & $E\{s\}$ & 0.167 \\
\hline $\max \{h\}$ & $\mathbf{0 . 2 0 9}$ & $\delta_{|2|}^{b}$ & $\mathbf{0 . 2 1 6}$ & $\gamma_{s}$ & 0.119 \\
\hline $\min \{h\}$ & 0.179 & HF & $\mathbf{- 0 . 2 1 6}$ & $\sigma\{s\}$ & -0.119 \\
\hline \hline
\end{tabular}

Within the HR signal extracted features, significant correlations are observed between average and maximum HR and reported entertainment preferences (see Table 11). These effects are consistent with the significant correlations of both $E\{h\}$ and $\max \{h\}$ on physiological data obtained from previous experiments using the Bug-Smasher game 814. Within the class of features extracted from the BVP signal, significant effects are observed on the mean of the absolute values of both the first and the second differences of the raw signal $\left(\delta_{|1|}^{b}, \delta_{|2|}^{b}\right)$ and on the energy of the HF band. On the contrary no significant effect appears in the class of SC features.

Obtained effects demonstrate that the higher the $\delta_{|1|}^{b}$ and $\delta_{|2|}^{b}$ values, the steeper the BVP signal and the higher the expressed "fun" preferences of children. Moreover, the lower the energy of the HRV HF band - which is driven by respiration and appears to derive mainly from vagal activity [10] - the more children appear to be entertained. Specifically, the energy of the HF range, representing quicker changes in HR, is primarily due to parasympathetic activity of the heart which is decreased during mental or stress load 9]10. This derives the conclusion that high mental or stress load appear to be the main factors that guide a child to prefer a game variant more than another.

The obtained statistically significant effects assume a linear relation between the respective features and reported entertainment which may (or may not) provide insight into the appropriate set of features on which to build a successful non-linear model of reported entertainment using preference learning. However, no safe conclusion can be derived for the appropriate feature subset before the proposed methodology is applied.

\subsection{Best Feature Selection}

Given the 85 pairs of preferred/non-preferred game comparisons, ANNs are evolved by following the neuro-evolution approach presented in [3]. The data is 
partitioned into $2 / 3$ training and $1 / 3$ validation data subsets and the leave-oneout cross-validation technique is used to obtain the average classification performance of the ANNs. In an attempt to minimize the ANN's size, it was determined that ANN architectures with 10 hidden neurons, are capable of successfully obtaining solutions of high fitness. As observed from Table 2, there is some consistency between features linearly related to reported entertainment and features that predict entertainment preferences based on a non-linear function (ANN). More specifically, all five features that correlate highly with reported entertainment (see Table 1) appear in Table2, Moreover, one may notice that the five highest performing features are extracted from BVP with HF energy being the highest performing feature $(66.67 \%$; average of $71.43 \%, 67.86 \%, 60.71 \%)$. Given that the average performance of 30 randomly generated ANNs (10 for each validation set) is $48.80 \%$, the p-value for the HF energy performance to occur is 0.043 .

The single feature experiments suggest that collecting physiological signals beyond HR, such as BVP, may provide more meaningful features (e.g. HF, $\delta_{|1|}^{b}$, $\left.\delta_{|2|}^{b}\right)$ for capturing entertainment preferences of children in physical play. The best performance obtained equals $66.67 \%$, which appears to be rather low. However, the reported complexity of classifying emotions through physiological state [16], the augmented signal noise recorded during physical play (especially through the BVP sensor) and the binomial-distributed probability of this performance to occur at random (0.043) suggest that the evolved ANNs are successful predictors of children's reported entertainment preferences based on a single feature extracted from physiological state.

Table 2. The ten highest performing features ranked by cross-validation performance from left to right

\begin{tabular}{c||c|c|c|c|c|c|c|c|c|c}
\hline \hline Feature & $\mathrm{HF}$ & $\min \{b\}$ & $\mathrm{LF}$ & $\delta_{|1|}^{b}$ & $\delta_{|2|}^{b}$ & $\max \{h\}$ & $\sigma\{R R\}$ & $h_{\text {in }}$ & $E\{h\}$ & $\min \{h\}$ \\
\hline Performance & 66.67 & 63.10 & 63.10 & 63.10 & 61.90 & 60.71 & 60.71 & 60.71 & 59.52 & 59.52 \\
\hline \hline
\end{tabular}

\section{Conclusion}

This paper explored the interplay between physiological signals and children's entertainment preferences in physical play. More specifically, the quantitative impact of children's reported entertainment on HR, BVP and SC signal statistics was investigated through action games developed on the Playware playground. The statistical effects obtained from the survey experiment presented here provide some first insights for the physiology of entertainment. Higher average and maximum HR, steeper blood volume signals and quicker changes in HR appear to correlate with higher levels of reported entertainment in children of the age group examined. The single feature experiments projected the impact of HF energy of HRV on reported entertainment indicating that there appears to be increased mental and stress load in preferred games. 
However, more complete conclusions will be derived when multi-feature selection is applied and the non-linear function between the selected feature subset and subject's preferences on 'fun' is generated. Even though HRV frequency bands provide indications about the interplay between affect, reported entertainment and physical activity, additional physical activity control experiments (see [4) will be required to isolate elements of physiology that correspond solely to entertainment preferences.

The proposed approach can be used for adaptation of the game's entertainment features (challenge, curiosity) according to the player's individual playing and physiological features in real-time in physical games. The key to this is the observation that the models (e.g. ANNs) relate features to an entertainment value. It is therefore possible in principle to infer what changes to game features (given embedding of the features in the model) will cause an increase in the entertainment value of the game, and to adjust game parameters to make those changes. For further discussion on this future direction the reader may refer to $[12$.

\section{Acknowledgments}

This work was supported in part by the Danish Research Agency, Ministry of Science, Technology and Innovation (project no: 274-05-0511).

\section{References}

1. Yannakakis, G.N., Hallam, J.: Towards Capturing and Enhancing Entertainment in Computer Games. In: Antoniou, G., Potamias, G., Spyropoulos, C., Plexousakis, D. (eds.) SETN 2006. LNCS (LNAI), vol. 3955, pp. 432-442. Springer, Heidelberg (2006)

2. Yannakakis, G.N., Hallam, J.: Game and Player Feature Selection for Entertainment Capture. In: Proceedings of the IEEE Symposium on Computational Intelligence and Games, pp. 244-251. IEEE Computer Society Press, Los Alamitos (2007)

3. Yannakakis, G.N., Hallam, J.: Preliminary Studies for Capturing Entertainment through Physiology in Physical Play. Technical Report TR-2007-5, Maersk Institue, University of Southern Denmark (2007)

4. Yannakakis, G.N., Hallam, J., Lund, H.H.: Entertainment Capture through Heart Rate Activity in Physical Interactive Playgrounds. User Modeling and UserAdapted Interaction, Special Issue: User Modeling and Affective Computing (to appear, 2007)

5. Mandryk, R.L., Inkpen, K.M., Calvert, T.W.: Using Psychophysiological Techniques to Measure User Experience with Entertainment Technologies. Behaviour and Information Technology (Special Issue on User Experience) 25, 141-158 (2006)

6. Zuckerman, M.: Sensation Seeking in Entertainment. In: Phychology of Entertainment, pp. 367-387. Lawrence Erlbaum Associates Publishers (2006)

7. Lund, H.H., Klitbo, T., Jessen, C.: Playware technology for physically activating play. Artifical Life and Robotics Journal 9, 165-174 (2005) 
8. Yannakakis, G.N., Hallam, J., Lund, H.H.: Capturing Entertainment through Heart-rate Dynamics in the Playware Playground. In: Harper, R., Rauterberg, M., Combetto, M. (eds.) ICEC 2006. LNCS, vol. 4161, pp. 314-317. Springer, Heidelberg (2006)

9. Rowe, D.W., Sibert, J., Irwin, D.: Heart Rate Variability: Indicator of User State as an aid to Human-Computer Interaction. In: Proceedings of Conference on Human Factors in Computing Systems, pp. 480-487 (1998)

10. Goldberger, J.J., Challapalli, S., Tung, R., Parker, M.A., Kadish, A.H.: Relationship of heart rate variability to parasympathetic effect. Circulation 103, 1977-1983 (2001)

11. Rani, P., Sarkar, N., Liu, C.: Maintaining optimal challenge in computer games through real-time physiological feedback. In: Proceedings of the $11^{\text {th }}$ International Conference on Human Computer Interaction (2005)

12. McQuiggan, S., Lee, S., Lester, J.: Predicting User Physiological Response for Interactive Environments: An Inductive Approach. In: Proceedings of the $2^{\text {nd }}$ Artificial Intelligence for Interactive Digital Entertainment Conference, pp. 60-65 (2006)

13. Ravaja, N., Saari, T., Turpeinen, M., Laarni, J., Salminen, M., Kivikangas, M.: Spatial Presence and Emotions during Video Game Playing: Does It Matter with Whom You Play? Presence Teleoperators \& Virtual Environments 15, 381-392 (2006 in press)

14. Hazlett, R.L.: Measuring emotional valence during interactive experiences: boys at video game play. In: CHI '06: Proceedings of the SIGCHI conference on Human Factors in computing systems, pp. 1023-1026. ACM Press, New York (2006)

15. Rosenthal, R.: Covert communication in laboratories, classrooms, and the truly real world. Current Directions in Psychological Science 12, 151-154 (2003)

16. Picard, R.W., Vyzas, E., Healey, J.: Toward Machine Emotional Intelligence: Analysis of Affective Physiological State. IEEE Trans. Pattern Anal. Mach. Intell. 23, 1175-1191 (2001)

17. Doyle, J.: Prospects for preferences. Computational Intelligence 20, 111-136 (2004)

18. Fiechter, C.N., Rogers, S.: Learning subjective functions with large margins. In: ICML '00: Proceedings of the Seventeenth International Conference on Machine Learning, pp. 287-294. Morgan Kaufmann Publishers Inc., San Francisco, CA, USA (2000)

19. Pincus, S.M.: Approximate entropy as a measure of system complexity. Proc. Natl. Acad. Sci. 88, 2297-2301 (1991)

20. Goldberger, A.L., Amaral, L.A.N., Glass, L., Hausdorff, J.M., Ivanov, P.C., Mark, R.G., Mietus, J.E., Moody, G.B., Peng, C.K., Stanley, H.E.: PhysioBank, PhysioToolkit, and PhysioNet: Components of a new research resource for complex physiologic signals. Circulation 101, 215-220 (2000) 